\title{
An Up-to-Date Anti-Cancer Treatment Strategy Focusing on HIF-1 $\alpha$ Suppression: Its Application for Refractory Ovarian Cancer
}

\author{
Mariko Fujita $^{1}$, Masanori Yasuda ${ }^{2}$, Kanae Kitatani ${ }^{1}$, Masaki Miyazawa ${ }^{1}$, \\ Kenichi Hirabayashi ${ }^{1}$, Susumu Takekoshi ${ }^{1}$, Tetsuji Iida ${ }^{3}$, Takeshi Hirasawa ${ }^{3}$, \\ Masaru Murakami ${ }^{3}$, Mikio Mikami ${ }^{3}$, Isamu Ishiwata ${ }^{4}$, Michio Shimizu² and \\ R. Yoshiyuki Osamura ${ }^{1}$
}

\author{
${ }^{1}$ Department of Pathology, Tokai University School of Medicine, ${ }^{2}$ Department of Pathology, Saitama Medical University \\ International Medical Center, ${ }^{3}$ Department of Obstetrics and Gynecology, Tokai University School of Medicine and \\ ${ }^{4}$ Ishiwata Obstetrics and Gynecology Hospital
}

Received October 5, 2007; accepted October 26, 2007; published online December 15, 2007

\begin{abstract}
Hypoxia inducible factor-1 $\boldsymbol{\alpha}$ (HIF-1 $\boldsymbol{\alpha}$ ) predominantly determines the transcriptional activity of HIF-1, which induces the certain genetic expressions to participate in the proliferation and progression of the tumor. It is supposed that HIF-1 $\alpha$ is also an extremely important factor in cancer treatment. Based on the results of our recent analyses using ovarian tumors, which indicated the close association of HIF- $1 \alpha$ expression with the acquisition of malignancy and the characterization of histology, we further investigated the possibility of a new strategy of cancer therapy that targeted HIF-1 $\alpha$ inhibition in the ovarian carcinoma. The cell line HUOCA-II, which originates from the refractory ovarian clear cell adenocarcinoma, was treated with rapamycin. The inhibitory effect of HIF-1 $\alpha$ was analyzed by immunohistochemistry and western blotting. It was demonstrated that inhibition of HIF-1 $\alpha$ and vascular endothelial growth factor (VEGF) expressions would lead to the down-regulation of tumor cell proliferation. Interestingly, there was little or no change in GLUT-1 expression by rapamycin administration. Thus, the inhibition of GLUT-1 may also be a key for the new strategy of cancer therapy as well as HIF-1 $\alpha$ and VEGF.
\end{abstract}

Key words: HIF-1 $\alpha$, GLUT-1, VEGF, rapamycin, clear cell adenocarcinoma

All normal and neoplastic tissues are thought to possess a mechanism to survive in a hypoxic condition by modulating certain crucial genes. Among them is hypoxia inducible factor-1 (HIF-1), which has been known as a key gene to adapt cells to microenvironmental conditions by up-regulation of transcription response to hypoxia [19] (Fig. 1). Thus, throughout the HIF-1-mediated pathway, various hypoxiarelated factors (HRFs) represented by vascular endothelial growth factor (VEGF) and glucose transporter-1 (GLUT-1) are activated [17]. HIF-1 is a heterodimeric protein consisting of an $\alpha$ subunit and a $\beta$ subunit, both of which contain a basic helix-loop-helix and PER-ARNT-SIM homology

Correspondence to: Masanori Yasuda, M.D., Department of Pathology, Saitama Medical University International Medical Center, 1397-1 Yamane, Hidaka, Saitama 350-1298, Japan.

E-mail:m_yasuda@saitama-med.ac.jp domains. While HIF-1 $\beta$ is constitutively expressed in the nucleus, HIF-1 $\alpha$ is known to be overexpressed in accordance with the hypoxic status. Under the non-hypoxic condition, HIF-1 $\alpha$ protein level is controlled by hydroxylation mediated by prolyl hydroxylase, and subsequently modified due to its binding to von Hippel-Lindau protein, finally leading to its degradation by the ubiquitin-proteasome system [ 8 , 14]. The adaptation of tumors to the hypoxic condition by efficient induction of HRFs is believed to contribute to their aggressiveness or poor prognosis, because these tumors achieve an increased resistance to chemotherapy and radiotherapy [11].

Overexpression of HIF-1 $\alpha$ could be involved as an early event of carcinogenesis in breast and prostate cancer [11]. There have been some positive links between the poor clinical outcome or increased mortality and strong expression of HIF-1 $\alpha$ [2-4, 21, 24]. In our study using ovarian 


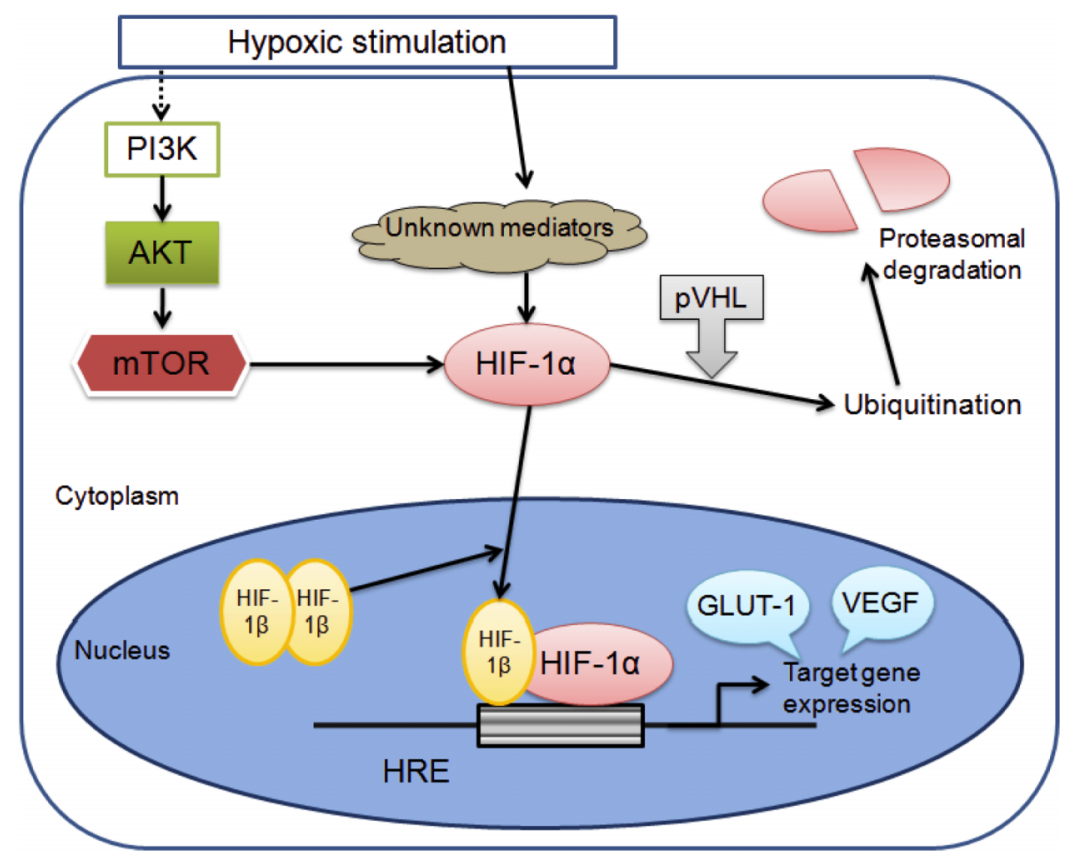

Fig. 1. HIF-1 $\alpha$ up-regulation and ubiquitination pathway. Mammalian target of rapamycin (mTOR) is a serine-threonine kinase that functions downstream of the phosphoinositide-3-kinase (PI3K) and AKT signaling cascade, and can be expected to be suppressed by certain agents such as rapamycin. The suppression effect on mTOR leads to down-regulation of hypoxia-related factors represented by GLUT-1 and VEGF.

tumors, immunohistochemical HIF-1 $\alpha$ expression tended to be strengthened in adenocarcinomas compared to adenomas and borderline tumors. As for the adenocarcinomas, interestingly enough, the expression profiles of HIF-1 $\alpha$ differed to some extent among each of the histologic types [23]. That is, the hypoxic status is expected to be closely related to the histologic feature such as papillary or piling structure accompanied by little or no vascular stroma, which is more commonly observed in the serous adenocarcinoma and clear cell adenocarcinoma [23]. This phenomenon was also satisfactorily explained by GLUT-1 overexpression in the thyroid papillary carcinoma [22]. The quantitative analysis of HIF-1 transcription activity using the ELISA technique corresponded with the immunohistochemical profiles: the highest value was noted in clear cell adenocarcinoma (data not shown). Lee et al. also reported that the hypoxia may change according to the histological type of ovarian cancer and that high HIF-1 $\alpha$ expression in the clear cell adenocarcinoma may confer chemoresistance [12]. Just recently, Osada et al. suggested that nuclear expression of HIF-1 $\alpha$ is a determinant prognostic factor of the ovarian carcinomas [16].

It should be noted that the interest in HIF-1 as a therapeutic target for malignant tumors has expanded exponentially in the last two decades [18]. Because suppression of HIF-1 could lead to a down-regulation of hypoxia-inducible genes, several kinds of anti-neoplastic/anti-angiogenic agents including nonsteroidal anti-inflammatory drugs have been utilized in experimental or clinical trials to clarify how effective they could be in the suppression of the activity and accumulation of HIF-1 $\alpha$ in some tumors [11]. Rapamycin, a macrolide antibiotic, is expected to inhibit the mammalian target of rapamycin (mTOR), which is a serine-threonine kinase that functions downstream of the phosphoinositide3-kinase-related kinase family [18] (Fig. 1). The recently exploited analogs (derivatives) of rapamycin were applied to experimental studies to investigate the efficacy as anti-tumor drugs as well as that as immunosuppressive drugs [5]. The analogs were also being clinically tested in patients with some tumors [1]. Using the experimental models to test the efficacy of rapamycin in ovarian cancer treatment, a significant correlation between HIF-1 $\alpha$ inhibition and VEGF down-regulation or increase of apoptosis has been demonstrated [9], and it was mentioned that rapamycin delays the tumor onset and progression [13]. Additional effects were found to be exerted when rapamycin is administered in combination with paclitaxel [9] and tamoxifen [20].

Our study was preliminarily designed to clarify whether the cell line from refractory ovarian cancer could be suppressed by administration of rapamycin alone. The cell line HUOCA-II, which was established from the recurrent ovarian clear cell adenocarcinoma after surgery and chemotherapy, was cultured in Ham's F-12 medium supplemented with $15 \%$ heat-inactivated fetal bovine serum (FBS; Gibco BRL, Grant Island, NY) and penicillin-streptomycin (Gibco BRL, Grant Island, NY) in a $5 \% \mathrm{CO}_{2}$ incubator at $37^{\circ} \mathrm{C}$. The cells treated with rapamycin (Sigma, St. Louis, MO) at a density of $100 \mathrm{nM}$ and $1 \mu \mathrm{M}$ were cultured for 6 hours, and then collected to make samples for immunohistochemical and western blotting analyses (HIF-1 $\alpha$ for immunohistochemistry, clone $\mathrm{H} 1 \alpha 67$, diluted at 1:60, Novus Biologicals, Littleton, CO; HIF-1 $\alpha$ for western blotting, 
polyclonal, diluted at 1:200, Santa Cruz Biotechnology, Santa Cruz, CA; VEGF, polyclonal, diluted at 1:50, Santa Cruz Biotechnology, Santa Cruz, CA; GLUT-1, polyclonal, diluted at 1:50, Dako, Carpinteria, CA). The differences in expression of HIF-1 $\alpha$, VEGF and GLUT-1 were semiquantitatively compared between rapamycin-treated cells and non-treated cells. HIF-1 $\alpha$ and VEGF expressions were prominently inhibited in rapamycin-treated cells; the inhibition was found to be dependent on the concentration of rapamycin. Interestingly, there was little or no change in GLUT-1 expression (Figs. 2, 3). The proliferation activity was evaluated by the labeling index of Ki-67 (clone MIB1, diluted at 1:50, Dako, Carpinteria, CA). Compared to non-treated cells, the index tended to decrease with the concentration-dependency as follows: non-treated, $31.7 \%$; $100 \mathrm{nM}, 27.2 \% ; 1 \mu \mathrm{M}, 22.4 \%$.

The results of our study were basically in concordance with those of recent studies in that HIF-1 $\alpha$ expression and
VEGF expression are closely linked [9]. But, to our knowledge, there have been no reports referring to the interaction between HIF-1 $\alpha$ and GLUT-1. We suppose that it may be difficult to suppress GLUT-1 expression by using rapamycin alone. GLUT-1 expression in ovarian tumors is considered to be related to malignant transformation [6], tumor invasion [10] and histological difference [23]. The overexpression of GLUT-1 in ovarian adenocarcinoma is commonly observed around areas which are far away from vascular vessels, suggesting GLUT-1 being a reliable marker of ischemia [7, 23]. Namely, GLUT-1 is expected to be essential for the tumor cells to survive especially in a severely hypoxic microenvironment in addition to HIF-1 $\alpha$. There has been only one report that suggests the possibility of GLUT-1-targeted anti-cancer therapy, which describes that the expression of antisense GLUT-1 mRNA via gene therapy can be used as a tool in the treatment of cancer [15]. Based on the hypothesis that GLUT-1 is also a key gene of anti-cancer therapy as
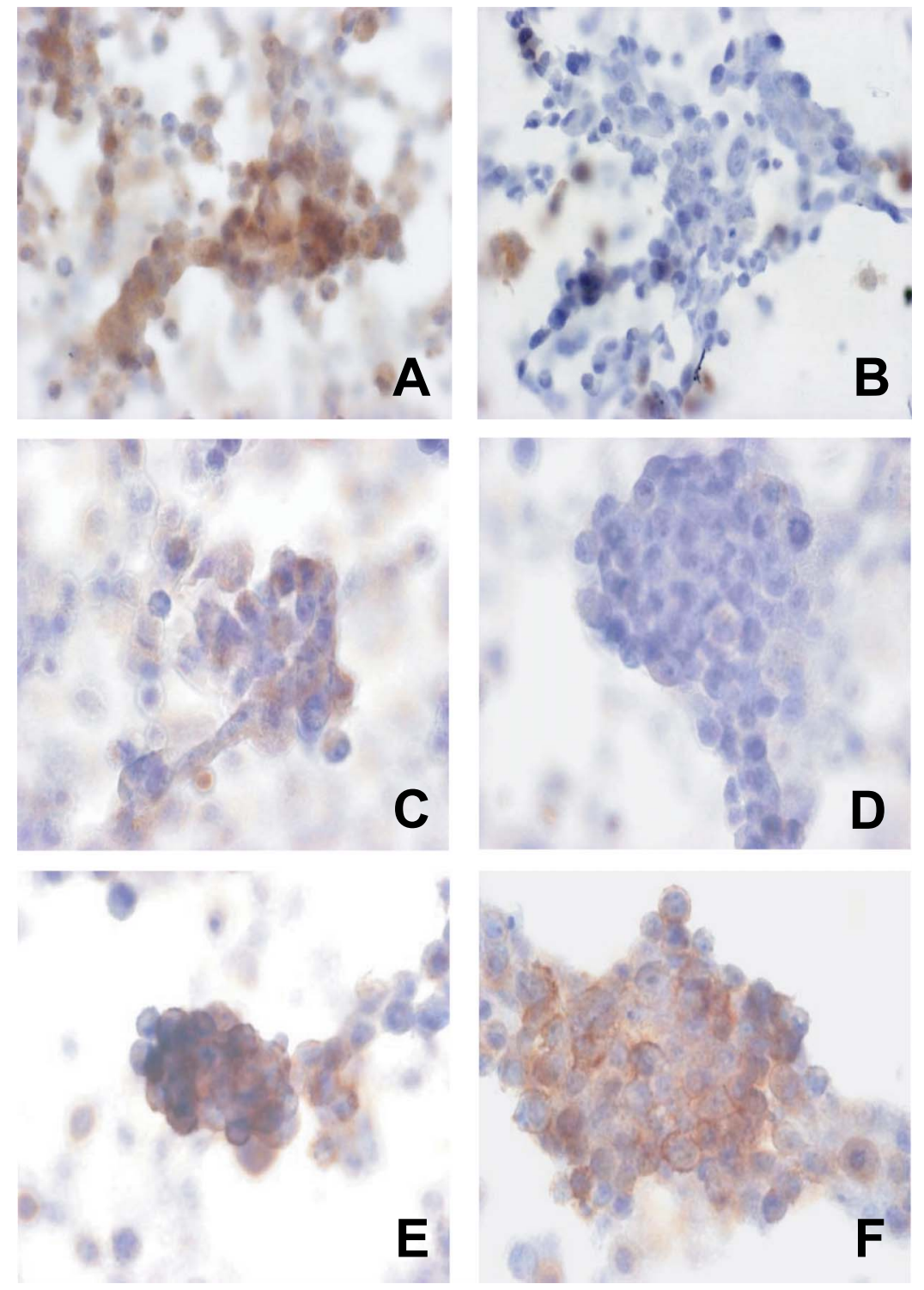

Fig. 2. Immunohistochemical expressions of HIF-1 $\alpha(\mathbf{A}, \mathbf{B})$, VEGF (C, D) and GLUT-1 (E, F). A, C, E: non-treated (negative control), $\mathbf{B}$, D, F: rapamycin-treated at $1 \mu \mathrm{M}$. In the cells treated with rapamycin, the positive reaction was considerably attenuated or completely suppressed for HIF-1 $\alpha$ and VEGF, but no apparent change in GLUT-1 expression was noted. 


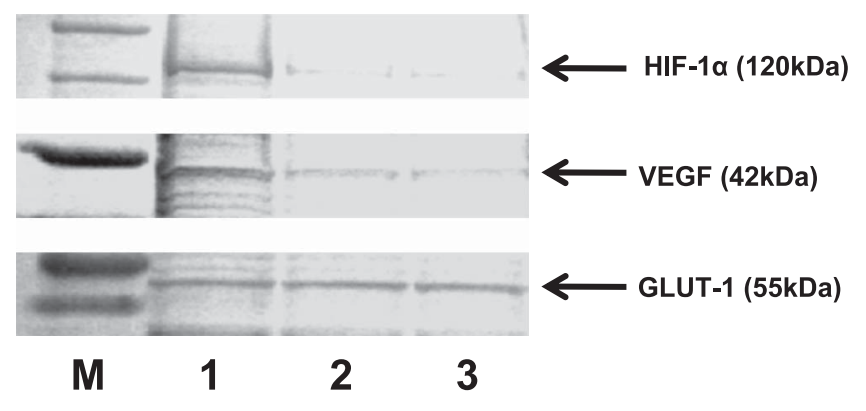

Fig. 3. Western blotting analysis. M: marker, 1: non-treated, 2: rapamycin-treated at $100 \mathrm{nM}, 3$ : rapamycin-treated at $1 \mu \mathrm{M}$. The decrease in positive reaction of HIF-1 $\alpha$ and VEGF showed a tendency toward concentration-dependency, but GLUT-1 expression was found to be unchangeable.

well as HIF-1 $\alpha$, we are working on a study to clarify the condition when more significant effects on tumor suppression by rapamycin can be achieved in vivo or in vitro in combination with the certain chemotherapeutic agents.

\section{References}

1. Atkins, M. B., Hidalgo, M., Stadler, W. M., Logan, T. F., Dutcher, J. P., Hudes, G. R., Park, Y., Liou, S. H., Marshall, B., Boni, J. P., Dukart, G. and Sherman, M. L. (2004) Randomized phase II study of multiple dose levels of CCI-779, a novel mammalian target of rapamycin kinase inhibitor, in patients with advanced refractory renal cell carcinoma. J. Clin. Oncol. 22; 909-918.

2. Birner, P., Schindl, M., Obermair, A., Plank, C., Breitenecker, G. and Oberhuber, G. (2000) Overexpression of hypoxia-inducible factor 1alpha is a marker for an unfavorable prognosis in earlystage invasive cervical cancer. Cancer Res. 60; 4693-4696.

3. Birner, P., Schindl, M., Obermair, A., Breitenecker, G. and Oberhuber, G. (2001) Expression of hypoxia-inducible factor 1alpha in epithelial ovarian tumors: its impact on prognosis and on response to chemotherapy. Clin. Cancer Res. 7; 1661-1668.

4. Bos, R., Zhong, H., Hanrahan, C. F., Mommers, E. C., Semenza G. L., Pinedo, H. M., Abeloff, M. D., Simons, J. W., van Diest, P. J. and van der Wall, E. (2001) Levels of hypoxia-inducible factor1 alpha during breast carcinogenesis. J. Natl. Cancer Inst. 93; 309-314.

5. Boulay, A., Rudloff, J., Zumstein-Mecker, S., O'Reilly, T., Evans, D. B., Chen, S. and Lane, H. A. (2005) Dual inhibition of mTOR and estrogen receptor signaling in vitro induces cell death in models of breast cancer. Clin. Cancer Res. 11; 5319-5328.

6. Cantuaria, G., Magalhaes, A., Penalver, M., Angioli, R., Braunschweiger, P., Gomez-Marin, O., Kanhoush, R., GomezFernandez, C. and Nadji, M. (2000) Expression of GLUT-1 glucose transporter in borderline and malignant epithelial tumors of the ovary. Gynecol. Oncol. 79; 33-37.

7. Cantuaria, G., Fagotti, A., Ferrandina, G., Magalhaes, A., Nadji, M., Angioli, R., Penalver, M., Mancuso, S. and Scambia, G. (2001) GLUT-1 expression in ovarian carcinoma: association with survival and response to chemotherapy. Cancer 92; 11441150.

8. Ikeda, E. (2005) Cellular response to tissue hypoxia and its involvement in disease progression. Pathol. Int. 55; 603-610.

9. Jiang, H. and Feng, Y. (2006) Hypoxia-inducible factor 1alpha (HIF-1alpha) correlated with tumor growth and apoptosis in ovarian cancer. Int. J. Gynecol. Cancer 1; 405-412.
10. Kalir, T., Wang, B. Y., Goldfischer, M., Haber, R. S., Reder, I., Demopoulos, R., Cohen, C. J. and Burstein, D. E. (2002) Immunohistochemical staining of GLUT1 in benign, borderline, and malignant ovarian epithelia. Cancer 94; 1078-1082.

11. Kimbro, K. S. and Simons, J. W. (2006) Hypoxia-inducible factor-1 in human breast and prostate cancer. Endocr. Relat. Cancer $13 ; 739-749$.

12. Lee, S., Garner, E. I., Welch, W. R., Berkowitz, R. S. and Mok, S. C. (2007) Over-expression of hypoxia-inducible factor 1 alpha in ovarian clear cell carcinoma. Gynecol. Oncol. 106; 311-317.

13. Mabuchi, S., Altomare, D. A., Connolly, D. C., Klein-Szanto, A., Litwin, S., Hoelzle, M. K., Hensley, H. H., Hamilton, T. C. and Testa, J. R. (2007) RAD001 (Everolimus) delays tumor onset and progression in a transgenic mouse model of ovarian cancer. Cancer Res. 67; 2408-2413.

14. Maxwell, P. H., Wiesener, M. S., Chang, G. W., Clifford, S. C., Vaux, E. C., Cockman, M. E., Wykoff, C. C., Pugh, C. W., Maher, E. R. and Ratcliffe, P. J. (1999) The tumour suppressor protein VHL targets hypoxia-inducible factors for oxygen-dependent proteolysis. Nature 399; 271-275.

15. Noguchi, Y., Saito, A., Miyagi, Y., Yamanaka, S., Marat, D., Doi, C., Yoshikawa, T., Tsuburaya, A., Ito, T. and Satoh, S. (2000) Suppression of facilitative glucose transporter $1 \mathrm{mRNA}$ can suppress tumor growth. Cancer Lett. 154; 175-182.

16. Osada, R., Horiuchi, A., Kikuchi, N., Yoshida, J., Hayashi, A., Ota, M., Katsuyama, Y., Mellilo, G. and Konishi, I. (2007) Expression of hypoxia-inducible factor lalpha, hypoxia-inducible factor 2alpha, and von Hippel-Lindau protein in epithelial ovarian neoplasms and allelic loss of von Hippel-Lindau gene: nuclear expression of hypoxia-inducible factor 1alpha is an independent prognostic factor in ovarian carcinoma. Hum. Pathol. 38; 13101320.

17. Semenza, G. L. (2002) HIF-1 and tumor progression: pathophysiology and therapeutics. Trends Mol. Med. 8; 62-67.

18. Semenza, G. L. (2003) Targeting HIF-1 for cancer therapy. Nat. Rev. Cancer 3; 721-732.

19. Semenza, G. L. and Wang, G. L. (1992) A nuclear factor induced by hypoxia via de novo protein synthesis binds to the human erythropoietin gene enhancer at a site required for transcriptional activation. Mol. Cell Biol. 12; 5447-5454.

20. Treek, O., Wackwitz, B., Haus, U. and Ortmann, O. (2006) Effects of a combined treatment with mTOR inhibitor RAD001 and tamoxifen in vitro on growth and apoptosis of human cancer cells. Gynecol. Oncol. 102; 292-299.

21. Wong, C., Wellman, T. L. and Lounsbury, K. M. (2003) VEGF and HIF-1alpha expression are increased in advanced stages of epithelial ovarian cancer. Gynecol. Oncol. 91; 513-517.

22. Yasuda, M., Ogane, N., Hayashi, H., Miyagi, Y., Iida, T., Mori, Y., Tsukinoki, K., Minematsu, T. and Osamura, Y. (2005) Glucose transporter-1 expression in the thyroid gland: clinicopathological significance for papillary carcinoma. Oncol. Rep. 14; 1499-1504.

23. Yasuda, M., Miyazawa, M., Fujita, M., Kajiwara, H., Iida, T., Hirasawa, T., Muramatsu, T., Murakami, M., Mikami, M., Saitoh, K., Shimizu, M., Takekoshi, S. and Osamura, R. Y. (2007) Expression of hypoxia inducible factor- $1 \alpha$ (HIF-1 $\alpha)$ and glucose transporter-1 (GLUT-1) in ovarian adenocarcinomas: Difference in hypoxic status depending in histological character. Oncol. Rep. (in press).

24. Zagzag, D., Zhong, H., Scalzitti, J. M., Laughner, E., Simons, J. W. and Semenza, G. L. (2000) Expression of hypoxia-inducible factor 1alpha in brain tumors: association with angiogenesis, invasion, and progression. Cancer 88; 2606-2618.

This is an open access article distributed under the Creative Commons Attribution License, which permits unrestricted use, distribution, and reproduction in any medium, provided the original work is properly cited. 